\title{
Sistema de Validação Automático de Sensores e AtuAdores
}

\author{
André Luis Furlanetti Gouvêa, Alexsandro da Silva, Alexandre Maria,
} Priscílio Roberto de Almeida e Bruno Michel Araújo da Silva

Magneti Marelli Indústria Comércio Automotivo Ltda.

E-mails: andre.gouvea@magnetimarelli.com, Alexsandro.silva@magnetimarelli.com, alexandre.maria@magnetimarelli.com, priscilio.almeida@magnetimarelli.com e bruno.silva@magnetimarelli.com

\section{RESUMO}

O desenvolvimento de um sistema de injeção para um veículo é composto de diversas fases dentre as quais podemos destacar algumas atividades como definição do mercado e do público alvo para a qual o motor e veículo serão disponibilizados, as normas de emissões de poluentes nacionais ou internacionais que devem ser respeitadas, e quais dispositivos eletrônicos e recursos de software de controle serão utilizados.

Após estas primeiras fases, iniciam-se as atividades de calibração dos mapas de funcionamento de motor, mapas de dirigibilidade do veículo, emissões, conforto, etc. E para garantir o funcionamento de todo o sistema também devem ser calibradas as funções de segurança e de diagnose elétrica. Quão mais rápido cada atividade possa ser executada, mais eficiente se torna o processo do desenvolvimento.

Este trabalho foi focado no aprimoramento da última atividade citada, a calibração e validação das funções de diagnose elétrica, que outrora eram feitas manualmente, repetidas vezes, gerando resultados diversos. Agora, embora ainda realizados repetidas vezes, os testes de validação podem ser realizados de forma automatizada, gerando resultados padronizados em um tempo consideravelmente menor.

\section{INTRODUÇÃO}

A indústria automobilística está em uma constante corrida atrás de novos desenvolvimentos, buscando sempre manter qualidade, propor inovações, atendendo as mais novas e rigorosas normas de restrições de emissões de poluentes e garantindo a melhor relação custo-benefício.

Nesta corrida, o sucesso de um produto, entre outros fatores está relacionado ao tempo que a montadora leva para disponibilizá-lo para o mercado. Para reduzir este tempo, as empresas de desenvolvimentos têm investido muito em modelamentos matemáticos, simulações, automatizações e virtualizações das atividades. Este trabalho apresenta, seguindo esta tendência, um aumento na eficiência do processo de calibração e validação das diagnoses elétricas, garantindo qualidade, repetibilidade e uma grande redução do tempo necessário para esta atividade através de uma proposta de um sistema automatizado. 


\section{DIAGNOSE ELÉTRICA - O ESTADO DA ARTE}

No princípio, os veículos utilizavam soluções eletromecânicas para todas as demandas do funcionamento normal (figura 01 e 02), e não haviam quaisquer monitoramentos ou quaisquer estratégias de recoveries para caso de um não funcionamento... Com o passar do tempo, para obter uma melhor performance, componentes e circuitos eletrônicos passaram a ser utilizados em conjunto com softwares de controle.

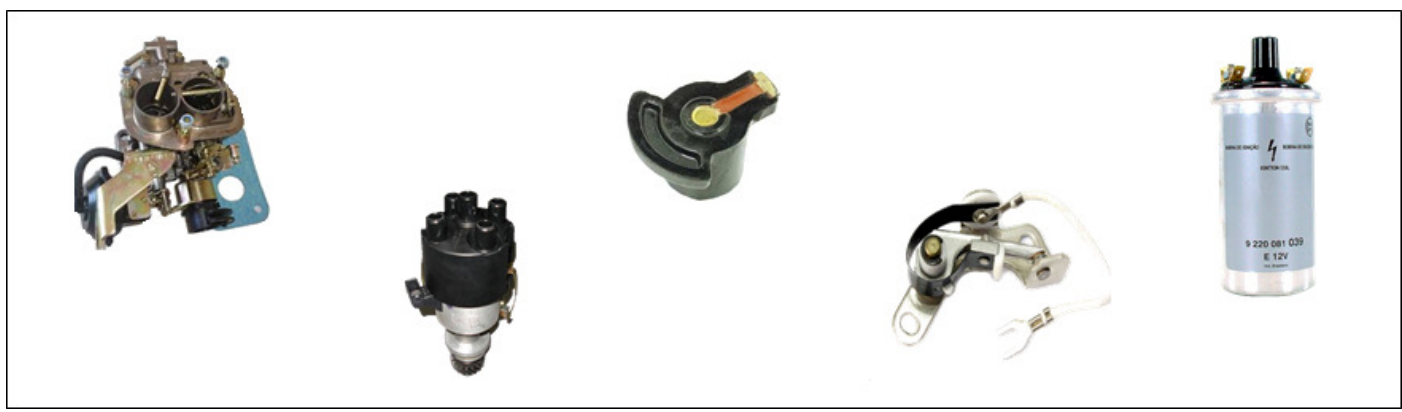

Figura 01 - dispositivos Eletromecânicos (da esquerda para direita: Carburador, distribuidor, rotor, platinado, bobina "single coil").

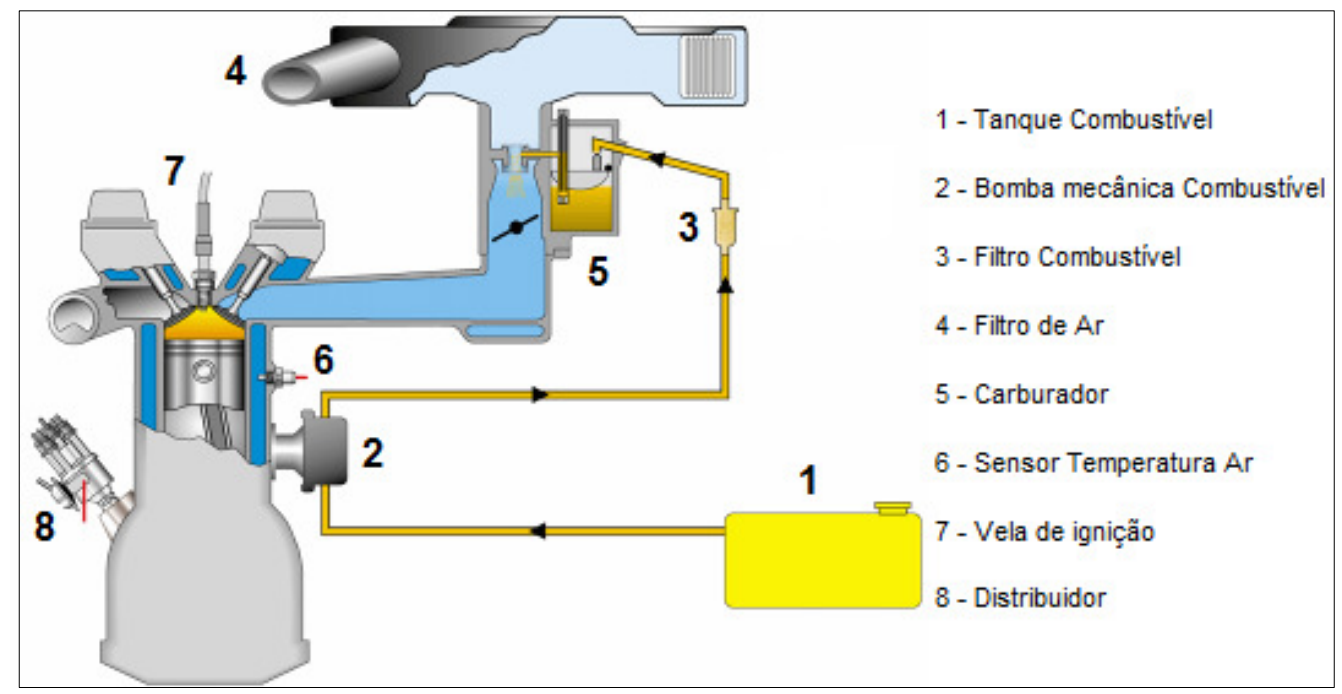

Figura 02 - Diagrama motor com soluções eletromecânicas.

Sistemas de injeção cada vez mais elaborados começaram a surgir, e a partir deste momento, para garantir o funcionamento do software de controle, tornou-se necessário monitorar o funcionamento dos componentes eletrônicos, (figura 03 e figura 04). Então funções de diagnose foram desenvolvidas para indicar quando algum dos componentes eletrônicos deixa de operar corretamente. Desta forma, todos os componentes relevantes para o funcionamento do sistema passaram a ser monitorados pelo software de controle.

Estes componentes auxiliam o sistema a trabalhar de maneira otimizada, levando em consideração altitude relativa, pressão do coletor, temperatura ambiente, etc. 


\section{$-\sqrt{2}$
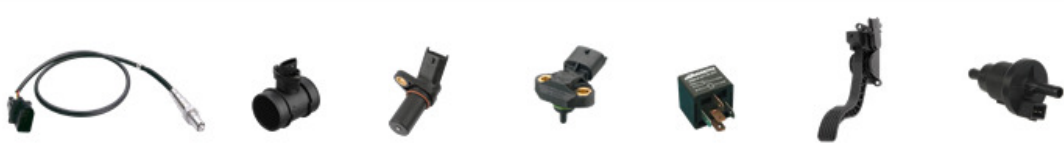

Figura 03 - exemplo de componentes utilizados sistema eletrônico veicular.

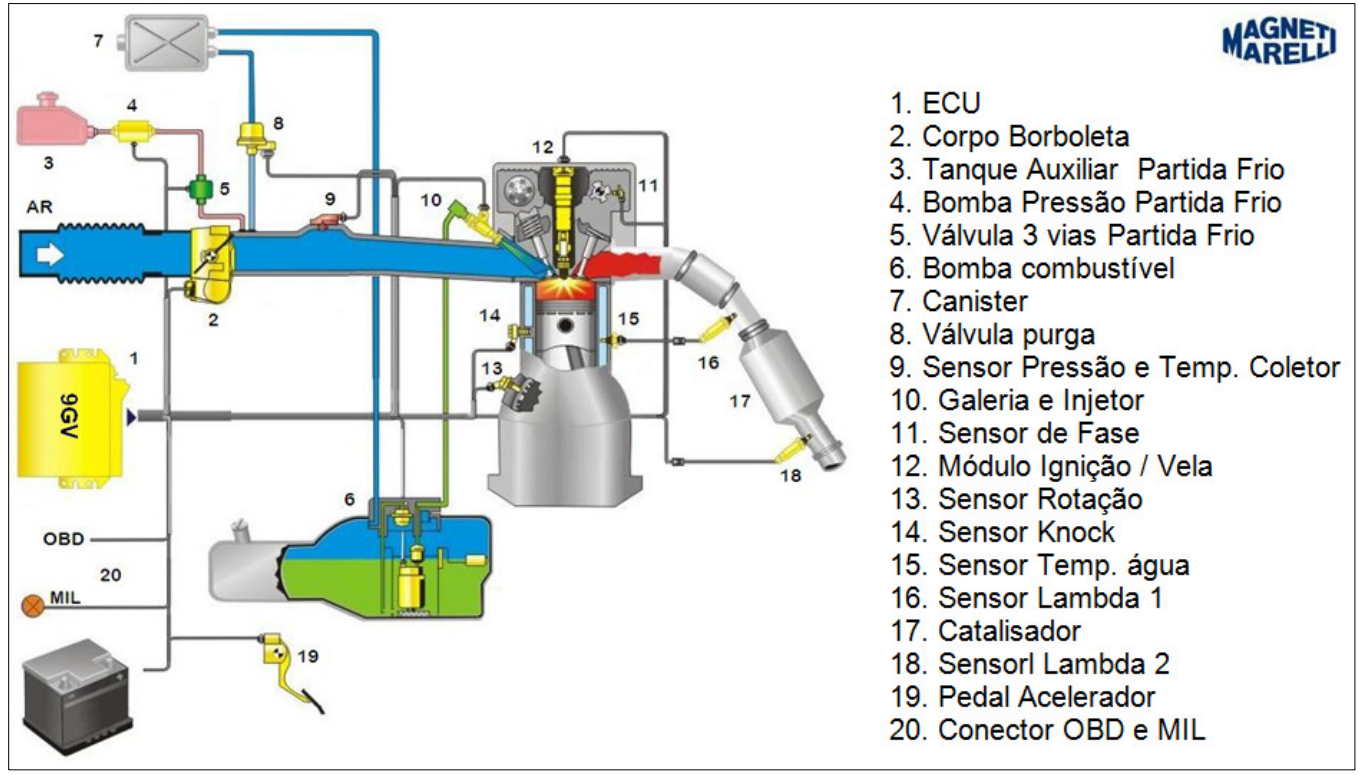

Figura 04 - Diagrama motor sistema injeção eletrônica

Os componentes eletrônicos conectados a unidade eletrônica do veículo (ECU) estão divididos em dois grupos distintos: Sensores (transdutores) e atuadores (figura 05).

\subsection{Sensores automotivos (Transdutores)}

Transdutores são dispositivos eletrônicos responsáveis pela conversão de grandezas físicas, tais como velocidade, temperatura, pressão, etc. em sinais elétricos. Os sinais destes transdutores são recebidos por entradas específicas da unidade eletrônica do veículo (ECU) quer sejam analógicos, frequênciais ou digitais, conforme o funcionamento de cada sensor.

Estes sinais são tratados dentro da unidade eletrônica do veículo (ECU) através de rotinas programáveis específicas de linearização e assim, são convertidos novamente nas grandezas físicas originais como temperatura, pressão, etc. Estes valores físicos alimentam os mapas de funcionamento de motor como os mapas de torque, de tempo de injeção, de eficiência volumétrica, de avanço, etc., que por sua vez acionam atuadores elétricos como injetores, motores de passo, corpo de borboleta e válvulas através de saídas digitais e de potência.

No momento em que é feita a linearização dos transdutores, também são definidos os valores máximos e mínimos toleráveis, considerando a dispersão na fabricação, envelhecimento ou desgaste do componente, funcionamento a frio ou a quente e tolerâncias da própria unidade eletrônica do veículo (ECU). Quando o sinal elétrico 
de um destes componentes ultrapassa estes limites calibrados, a rotina de identificação de falhas alerta o sistema do problema identificado.

\subsection{Atuadores eletrônicos}

Os atuadores são dispositivos eletrônicos capazes de exercer alguma atividade mecânica específica a partir de comandos elétricos da unidade eletrônica do veículo (ECU), por exemplo: Válvulas solenoides de controle de fluxo (injetores, canister, etc.), motores para movimentos lineares ou angulares (corpos de borboleta, controle do comando de válvulas variável, motores de passo etc.), ou simplesmente para promover contatos elétricos como o caso dos reles (utilizados para os aquecedores das sondas de oxigênio, bomba sistema combustível, iluminação, etc.).

Os atuadores estão conectados as saídas digitais ou saídas de potência da unidade eletrônica do veículo (ECU) que constantemente os monitora para o caso de curtoscircuitos ou rompimento da conexão. Em caso de algum destes eventos, a rotina de identificação de falhas também alerta o sistema do problema identificado.

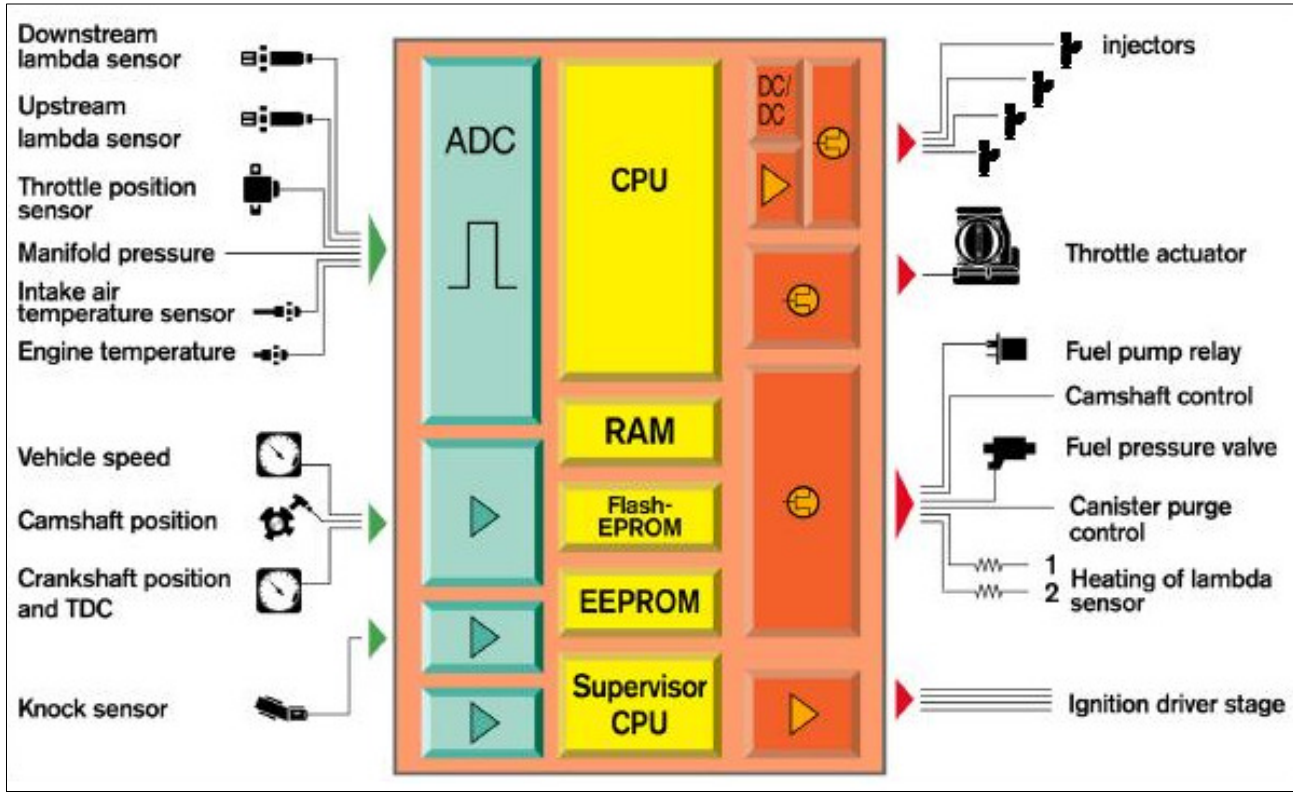

Figura 05 - exemplo de sensores (esquerda), ECU (central) e atuadores (direita)

\section{FUNCIONAMENTO DA DIAGNOSE ELÉTRICA}

A Detecção das falhas ocorre considerando eventos específicos de funcionamento do motor e do veículo. Para componentes exclusivamente elétrico-passivos ou ativos por fonte da unidade eletrônica do veículo (ECU), a detecção de falhas ocorre após a energização do sistema, ou seja, após chave de ignição ligada (exemplo: Sensores de temperatura, pressão, etc.). Para outros, no entanto elétrico-passivos por indução e ativos sem fonte interna, (fase, rotação, injetores, ignição, etc.), a detecção está condicionada ao funcionamento do motor. Para os que dependem de condições dinâmicas do sistema como carga, velocidade, longos períodos de desaceleração, (exemplo: falha funcional da sonda de oxigênio, sensor de velocidade, etc.), a detecção só ocorre quando o veículo estiver na condição específica. 
E conforme descrito, quando algum componente eletrônico (sensor ou atuador) apresentar um funcionamento anômalo, dentro da condição específica de funcionamento do motor ou veículo, a rotina de gerenciamento do tal componente inicia um processo de validação da falha, acionando as funções de monitoramentos. Contadores em função do tempo são inicializados para garantir que a detecção da falha não ocorra de forma errônea.

Quando os contadores atingem um valor pré-determinado, um log de erro é enviado ao gerenciamento do sistema de controle, que tomas as medidas cabíveis dentro das possibilidades existentes para cada função: Desconsideração dos valores medidos (caso a falha esteja em algum sensor - uma vez que este sensor está apresentando erro de leitura), utilização de valores calculados ou valores pré-determinados como medida de segurança, acionamento de sistemas de segurança que reduzem a potência do motor colocando-o fora de risco.

Caso a falha ocorra com algum atuador, o sistema avalia a possibilidade de continuar ou não o funcionamento do motor.

Também estão entre as decisões, o registro do erro encontrado na memória de falhas (acessível via ferramenta de diagnose) e acendimento da lâmpada que indica o mal funcionamento - MIL, (Figura 06).

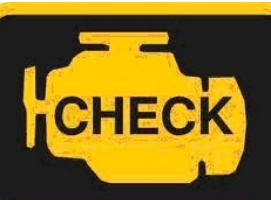

Figura 06 - Lâmpada indicativa de mal funcionamento

\section{PROCESSO ATUAL DE VALIDAÇÃO DA DIAGNOSE ELÉTRICA}

Desde o primórdios dos sistemas eletrônicos de injeção veicular, o processo de validação das funções de monitoramento é realizado utilizando uma unidade eletrônica do veículo com interface de desenvolvimento conectada ao laptop e ferramenta de diagnose para leitura da memória de falhas (Figura 07).

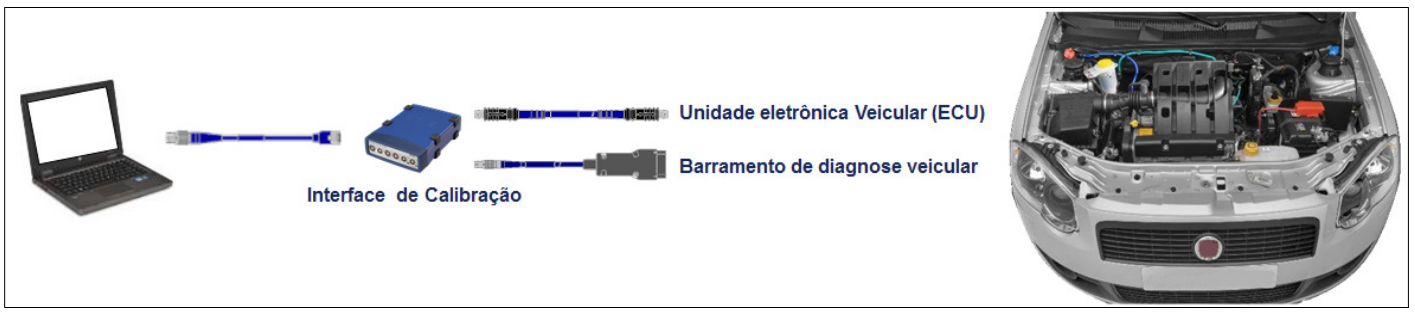

Figura 07 - Interface de desenvolvimento / validação sistema eletrônico de injeção.

Mas a simulação dos modos de falha é executado "manualmente", sensor por sensor, atuador por atuador, utilizando como instrumento gerador de falha, caixas de passagem (Figura 08), conhecidos como break out box, que fazem a interceptação dos sinais do veículo até a central 
através de bornes elétricos (onde é possível gerar curtos-circuitos) e chaves "liga-desliga" (para simular a desconexão do componente).

Estes equipamentos em sua maioria são artesanais (muitas vezes rudimentares), não possuem capacidade de nenhuma interação com as ferramentas de aquisição de dados, operada sempre de forma manual, não imune a ruídos e interferências externas, e limitada quanto aos modos de falha que pode gerar: Curto-circuito para positivo da bateria (12 Volts), curto-circuito para negativo da bateria ( 0 Volts), circuitos abertos e através de potenciômetros externos, simular erros falta de plausibilidade entre sinais conhecidos do sistema.

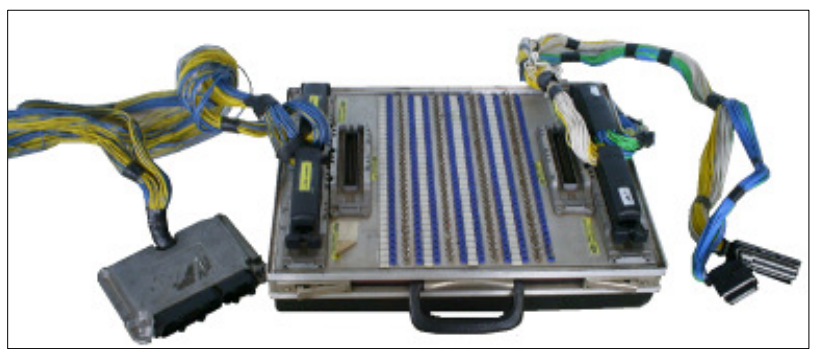

Figura 08 - Caixa de passagem "maleta".

Devido ao fato de ser totalmente operada de forma manual, os testes executados com este dispositivo de simulação de falhas, não podem ser realizados dentro um padrão, pois depende da agilidade do engenheiro validador.

Atendendo as normas que regulam as emissões dos gases do escapamento, muitos testes para serem validados devem ser executados repetidas vezes, garantindo uma quantidade de ciclos de funcionamento e desligamento do motor para que a lâmpada que indica o mal funcionamento (MIL) acenda e a falha testada seja gravada na memória da unidade eletrônica do veículo (ECU), assim como uma outra quantidade destes ciclos sem a falha para identificar a solução do problema.

Estas validações são necessárias durante praticamente todas as fases do desenvolvimento do sistema eletrônico, garantindo assim que nenhuma irregularidade será encontrada.

\section{PROPOSTA DO SISTEMA AUTOMATIZADO DE VALIDAÇÃO}

O sistema proposto tem dois principais objetivos: Interação das ferramentas utilizadas na atividade de validação das funções de monitoramento eletrônico e automatização do processo de validação.

\subsection{Interação das ferramentas}

Para integrar as diversas subatividades da validação das funções de monitoramento, vários dispositivos e ferramentas foram utilizados:

\subsubsection{Gerador eletrônico de Falhas}

Este dispositivo gerador de falhas eletrônico (Figura 09 e Figura 10 ), é capaz de gerar falhas em tempo real, com duração pré-definida ou com duty-cycle 
definido, sejam elas com comportamento intermitentes ou contínuos. Além dos modos de falha triviais (curtos-circuitos e circuitos abertos), este equipamento pode gerar falhas com impedâncias parasitas e corrente elétrica de fuga, que controlamos através de comandos de um programa dedicado instalado também no mesmo laptop que gerencia o software de controle da unidade eletrônica do veículo.

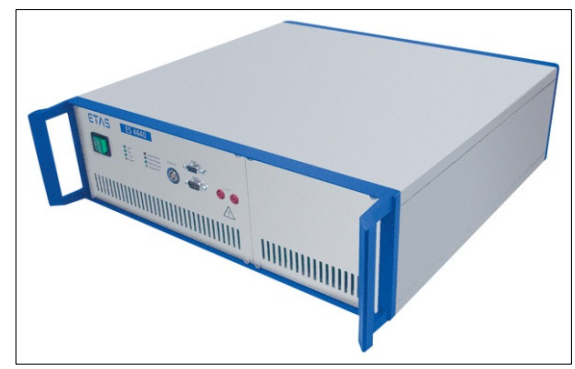

Figura 09 - Gerador eletrônico de Falhas

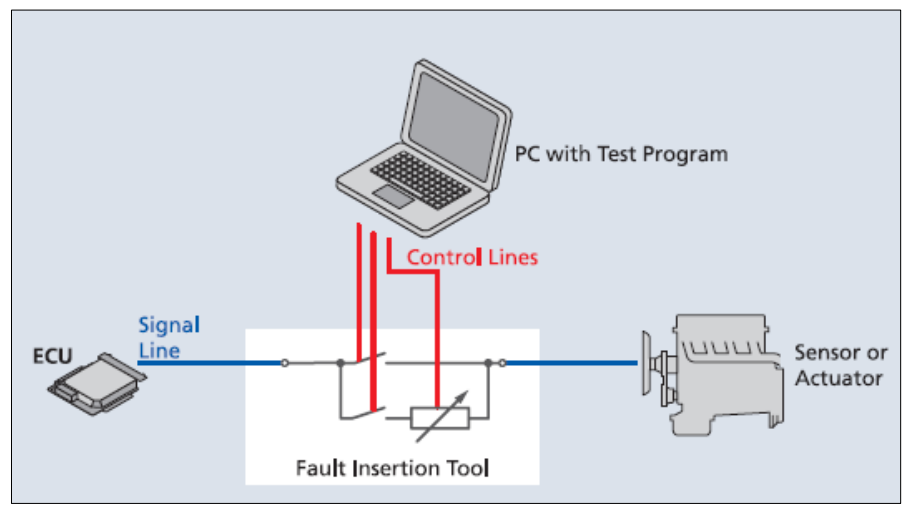

Figura 10 - Gerador eletrônico de Falhas

\subsubsection{Interface de controle elétrico}

A interface de controle elétrico através do software de controle da unidade eletrônica do veículo (ECU), nos possibilita envio de comando elétricos para controlar a chave de ignição e o motor de partida (Figuras 11).

Um display instalado no painel do veículo está conectado aos comandos primários dos veículo (chave de ignição e motor de partida) e nos permite monitorar o funcionamento da interface de controle elétrico, assim como a tensão que está sendo utilizada para simular o erro no componente eletrônico do veículo (Figura 12).

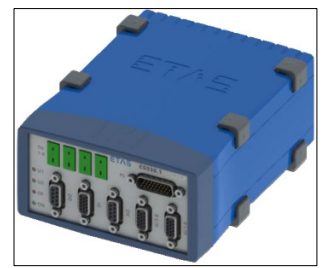

Figura 11 - Interface de controle elétrico 


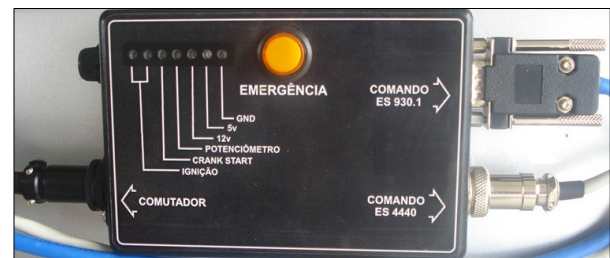

Figura 12 - Display Interface de controle elétrico.

\subsubsection{Software integrado de diagnose veicular $O B D$}

Outra novidade foi a utilização de um sistema integrado de leitura do barramento de diagnose veicular OBD, integrado ao software de controle da unidade eletrônica do veículo. Desta forma, não necessitamos da ferramenta externa para leitura da memória de falhas do veículo.

\subsection{Automatização do Processo de Validação}

Com os equipamentos e softwares supra citados montados no veículo em desenvolvimento (Figura 13), um algoritmo de programação que contempla todas as fases da atividade de validação foi escrito de forma a atender criteriosamente a atividade de teste e validação.

Desta forma, definida a sequência dos componentes que deverão ser testados, este script, inicializa o softwares de desenvolvimento da unidade eletrônica do veículo, assim como também os controles das interfaces de simulação de falhas e controle elétrico.

O sistema inicia o teste gerando um comando para ligar a chave de ignição, energizando o módulo eletrônico do veículo, inicia-se a aquisição das informações ambientais (temperaturas da água, do óleo, do ambiente, pressão de coletor, ângulo de abertura do corpo de borboleta, posição do pedal do acelerador, além de outros tantos dados disponíveis), e também das informações específicas de cada sensor ou atuador que será testado como sinais analógicos, limite para validação das falhas, contadores, etc..

Dependendo do tipo da validação ou da necessidade específica do componente a ser testado, o motor é ligado através da interface de controle eletrônico, então o simulador de falhas gera o modo de falha que deve ser testado (curto-circuito, circuito interrompido, etc.).

O script de validação da falha identifica quando o software de controle do veículo registra a falha e comanda o desligamento do veículo, assim como a nova partida do motor, até que as exigências normativas sejam cumpridas. Em seguida o erro é removido e repete-se o processo de ligar e desligar o motor conforme a necessidade.

Quando o erro já está validado, a memória de falhas do veículo é apagada e a aquisição salva automaticamente. Então o sistema já está apto para iniciar a próxima validação repetindo o processo. 


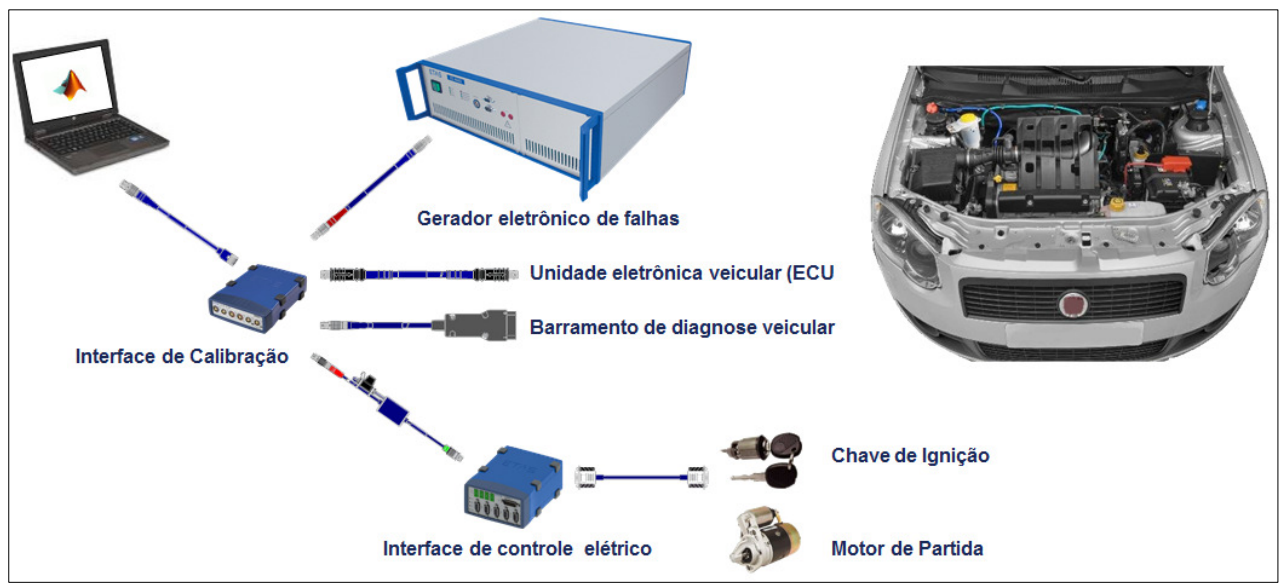

Figura 13 - Sistema automatizado de validação da diagnose elétrica

\section{CONCLUSÃO}

A utilização desta ferramenta automatizada, trouxe como benefício imediato, uma redução significativa do tempo de validação, aumento da gama dos modos de falha possíveis, utilização em qualquer sistema em pelo ao menos noventa por cento das funções de monitoramento dos componentes eletrônicos, a padronização dos métodos de aquisição, resultados mais confiáveis, dinamismo na confecção de relatórios e relatórios mais otimizados, possibilidade de se programar e executar diferentes rotinas de teste, conforme a necessidade (Figura 14).

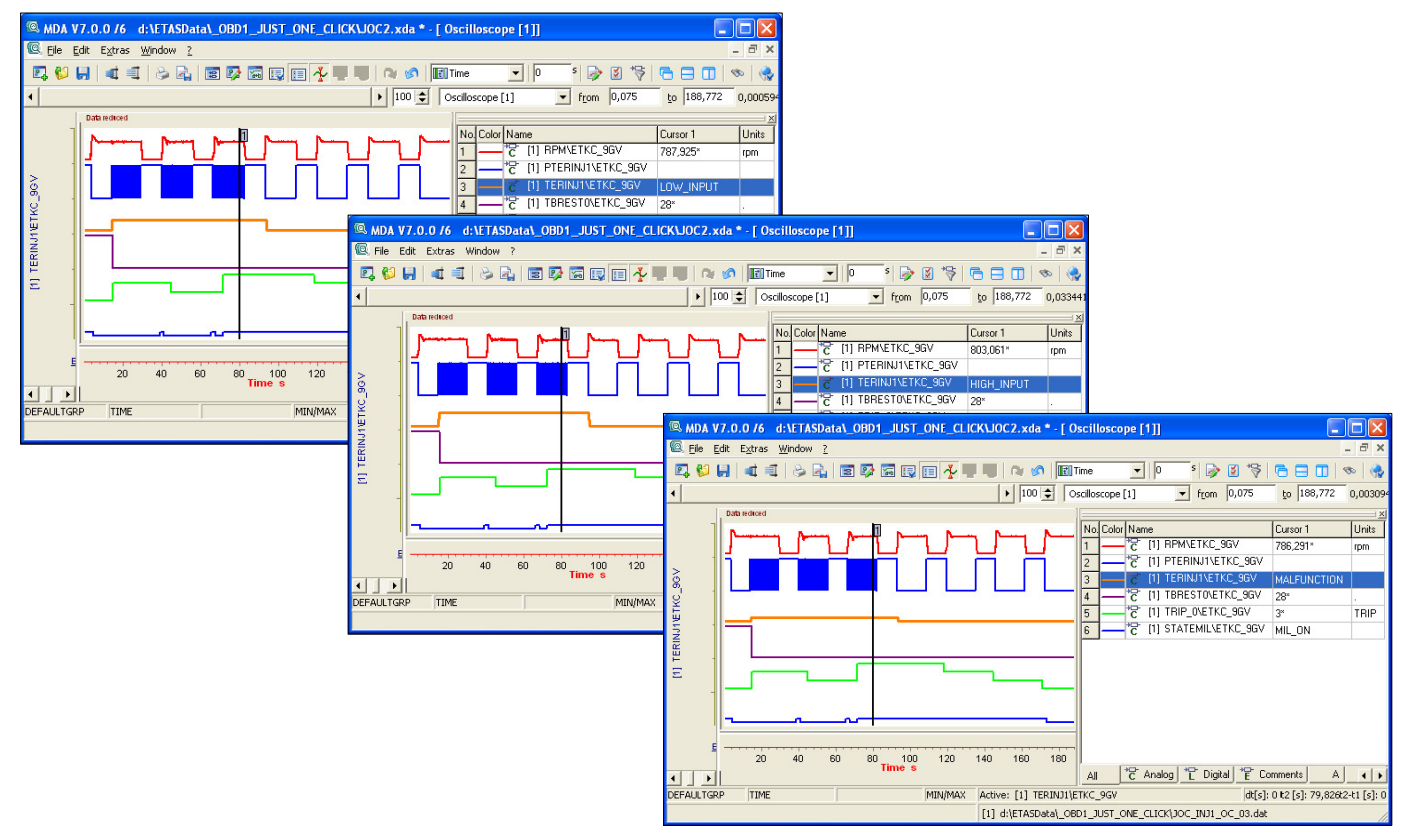

Figura 14 - Aquisição de dados de um atuador em três modos de falha diferentes: Curtocircuito para $0 \mathrm{~V}$, curto-circuito para $12 \mathrm{~V}$ e interrupção de circuito. 